\title{
Oscillation analysis for nonlinear difference equation with non-monotone arguments
}

\section{Özkan Öcalan ${ }^{\text {** }}$, Umut Mutlu Özkan² and Mustafa Kemal Yildiz²}

\section{"Correspondence:}

ozkanocalan@akdeniz.edu.tr

'Department of Mathematics,

Faculty of Science, Akdeniz

University, Antalya, Turkey

Full list of author information is

available at the end of the article

\begin{abstract}
The aim of this paper is to obtain some new oscillatory conditions for all solutions of nonlinear difference equation with non-monotone or non-decreasing argument

$$
\Delta x(n)+p(n) f(x(\tau(n)))=0, \quad n=0,1, \ldots
$$

where $(p(n))$ is a sequence of nonnegative real numbers and $(\tau(n))$ is a non-monotone or non-decreasing sequence, $f \in C(\mathbb{R}, \mathbb{R})$ and $x f(x)>0$ for $x \neq 0$.
\end{abstract}

MSC: $39 \mathrm{~A} 10$

Keywords: Delay difference equation; Non-monotone arguments; Nonlinear; Oscillation

\section{Introduction}

Oscillation theory of difference equations has attracted many researchers. In recent years there has been much research activity concerning the oscillation and nonoscillation of solutions of delay difference equations. For these oscillatory and nonoscillatory results, we refer, for instance, to [1-23]. As far as we can see, there is not yet a study in the literature about the solutions of Eq. (1) to be oscillatory under the $(\tau(n))$ is a non-monotone or non-decreasing sequence. So, in the present paper, our aim is to obtain new oscillatory conditions for all solutions of Eq. (1). Consider the nonlinear difference equation with general argument

$$
\Delta x(n)+p(n) f(x(\tau(n)))=0, \quad n=0,1, \ldots,
$$

where $(p(n))_{n \geq 0}$ is a sequence of nonnegative real numbers and $(\tau(n))_{n \geq 0}$ is a sequence of integers such that

$$
\tau(n) \leq n-1 \quad \text { for all } n \geq 0 \quad \text { and } \quad \lim _{n \rightarrow \infty} \tau(n)=\infty
$$

and

$$
f \in C(\mathbb{R}, \mathbb{R}) \quad \text { and } \quad x f(x)>0 \quad \text { for } x \neq 0 .
$$

(c) The Author(s) 2018. This article is distributed under the terms of the Creative Commons Attribution 4.0 International License (http://creativecommons.org/licenses/by/4.0/), which permits unrestricted use, distribution, and reproduction in any medium, provided you give appropriate credit to the original author(s) and the source, provide a link to the Creative Commons license, and indicate if changes were made. 
$\Delta$ denotes the forward difference operator $\Delta x(n)=x(n+1)-x(n)$.

Define

$$
r=-\min _{n \geq 0} \tau(n)
$$

Clearly, $r$ is a positive integer.

By a solution of the difference equation (1), we mean a sequence of real numbers $(x(n))_{n \geq-r}$ which satisfies (1) for all $n \geq 0$.

A solution $(x(n))_{n \geq-r}$ of the difference equation (1) is called oscillatory, if the terms $x(n)$ of the sequence are neither eventually positive nor eventually negative. Otherwise, the solution is said to be nonoscillatory.

If $f(x)=x$, then Eq. (1) takes the form

$$
\Delta x(n)+p(n) x(\tau(n))=0, \quad n=0,1, \ldots
$$

In particular, if we take $\tau(n)=n-\ell$, where $\ell>0$, then Eq. (4) reduces to

$$
\Delta x(n)+p(n) x(n-\ell)=0 .
$$

In 1989, Erbe and Zhang [8] proved that each one of the conditions

$$
\liminf _{n \rightarrow \infty} p(n)>\frac{\ell^{\ell}}{(\ell+1)^{\ell+1}}
$$

and

$$
\limsup _{n \rightarrow \infty} \sum_{j=n-\ell}^{n} p(j)>1
$$

is sufficient for all solutions of (5) to be oscillatory.

In the same year, 1989, Ladas, Philos and Sficas [12] established that all solutions of (5) are oscillatory if

$$
\liminf _{n \rightarrow \infty}\left[\frac{1}{\ell} \sum_{j=n-\ell}^{n-1} p(j)\right]>\frac{\ell^{\ell}}{(\ell+1)^{\ell+1}}
$$

Clearly, condition (7) improves to (5).

In 1991, Philos [15] extended the oscillation criterion (8) to the general case of Eq. (4), by establishing that, if the sequence $(\tau(n))_{n \geq 0}$ is increasing, then the condition

$$
\liminf _{n \rightarrow \infty}\left[\frac{1}{n-\tau(n)} \sum_{j=\tau(n)}^{n-1} p(j)\right]>\limsup _{n \rightarrow \infty} \frac{(n-\tau(n))^{n-\tau(n)}}{(n-\tau(n)+1)^{n-\tau(n)+1}}
$$

suffices for the oscillation of all solutions of Eq. (4).

In 1998, Zhang and Tian [20] found that if $(\tau(n))$ is non-decreasing,

$$
\lim _{n \rightarrow \infty}(n-\tau(n))=\infty
$$


and

$$
\liminf _{n \rightarrow \infty} \sum_{j=\tau(n)}^{n-1} p(j)>\frac{1}{\mathrm{e}}
$$

then all solutions of Eq. (4) are oscillatory.

Later, in 1998, Zhang and Tian [21] found that if $(\tau(n))$ is non-decreasing or nonmonotone,

$$
\limsup _{n \rightarrow \infty} p(n)>0
$$

and (10) holds, then all solutions of Eq. (4) are oscillatory.

In 2008, Chatzarakis, Koplatadze and Stavroulakis [3] proved that if $(\tau(n))$ is nondecreasing or non-monotone $h(n)=\max _{0 \leq s \leq n} \tau(s)$,

$$
\limsup _{n \rightarrow \infty} \sum_{j=\tau(n)}^{n} p(j)>1
$$

then all solutions of Eq. (3) are oscillatory.

In 2008, Chatzarakis, Koplatadze and Stavroulakis [4] proved that if $(\tau(n))$ is nondecreasing or non-monotone, $h(n)=\max _{0 \leq s \leq n} \tau(s)$,

$$
\limsup _{n \rightarrow \infty} \sum_{j=\tau(n)}^{n} p(j)<\infty
$$

and (10) holds, then all solutions of Eq. (4) are oscillatory.

In 2006, Yan, Meng and Yan [18] found that if $(\tau(n))$ is non-decreasing,

$$
\liminf _{n \rightarrow \infty} \sum_{j=\tau(n)}^{n-1} p(j)>0
$$

and

$$
\liminf _{n \rightarrow \infty} \sum_{j=\tau(n)}^{n-1} p(j)\left(\frac{j-\tau(j)+1}{j-\tau(j)}\right)^{j-\tau(j)+1}>1,
$$

then all solutions of Eq. (4) are oscillatory.

In 2016, Öcalan [16] proved that if $(\tau(n))$ is non-decreasing or non-monotone, $h(n)=$ $\max _{0 \leq s \leq n} \tau(s)$ and (16) holds, then all solutions of Eq. (4) are oscillatory.

Set

$$
k(n)=\left(\frac{n-\tau(n)+1}{n-\tau(n)}\right)^{n-\tau(n)+1}, \quad n \geq 1 .
$$

Clearly

$$
\mathrm{e} \leq k(n) \leq 4, \quad n \geq 1 .
$$


Observe that it is easy to see that

$$
\sum_{j=\tau(n)}^{n-1} p(j) k(j) \geq \mathrm{e} \sum_{j=\tau(n)}^{n-1} p(j)
$$

and therefore condition (16) is better than condition (11).

When the case $\tau(n)=n-\ell$, where $\ell>0$, then Eq. (1) reduces to

$$
\Delta x(n)+p(n) f(x(n-\ell))=0, \quad n=0,1, \ldots
$$

For Eq. (19), we can suggest references [11] and [17] for the reader.

\section{Main results}

In this section we investigated the oscillatory behavior of all solutions of Eq. (1). We present new sufficient conditions for the oscillation of all solutions of Eq. (1) under the assumption that the argument $(\tau(n))$ is non-monotone or non-decreasing sequence. Set

$$
h(n)=\max _{0 \leq s \leq n} \tau(s) .
$$

Clearly, $(h(n))$ is non-decreasing, and $\tau(n) \leq h(n)$ for all $n \geq 0$. We note that if $(\tau(n))$ is non-decreasing, then we have $\tau(n)=h(n)$ for all $n \geq 0$.

Assume that the $f$ in Eq. (1) satisfies the following condition:

$$
\limsup _{x \rightarrow 0} \frac{x}{f(x)}=M, \quad 0 \leq M<\infty
$$

Theorem 1 Assume that (2), (3) and (21) hold. If $(\tau(n))$ is non-monotone or nondecreasing, and

$$
\liminf _{n \rightarrow \infty} \sum_{j=\tau(n)}^{n-1} p(j)>\frac{M}{\mathrm{e}},
$$

then all solutions of Eq. (1) oscillate.

Proof Assume, for the sake of contradiction, that $(x(n))$ is an eventually positive solution of (1). Then there exists $n_{1} \geq n_{0}$ such that $x(n), x(\tau(n)), x(h(n))>0$ for all $n \geq n_{1}$. Thus, from Eq. (1) we have

$$
\Delta x(n)=-p(n) f(x(\tau(n))) \leq 0 \quad \text { for all } n \geq n_{1} .
$$

Thus $(x(n))$ is non-increasing and has a limit $k \geq 0$ as $n \rightarrow \infty$. Now, we claim that $k=0$. Otherwise, $k>0$. By (3), $f(x)>0$ and then $\lim _{n \rightarrow \infty} f(x(n))=f(k)>0$. So, summing up (1) from $n_{1}$ to $n-1$, we get

$$
x(n)=x\left(n_{1}\right)-\sum_{j=n_{1}}^{n-1} p(j) f(x(\tau(j))) .
$$


On the other hand, condition (22) implies that

$$
\sum_{j=n_{1}}^{\infty} p(j)=\infty
$$

In view of (23) and (24), we obtain for $n \rightarrow \infty$

$$
k=x\left(n_{1}\right)-f(k) \sum_{j=n_{1}}^{\infty} p(j)=-\infty
$$

This is a contradiction to the fact that $k>0$. Therefore $\lim _{n \rightarrow \infty} x(n)=0$. Now, suppose $M>0$. Then, in view of (21) we can choose $n_{2} \geq n_{1}$ so large that

$$
f(x(n)) \geq \frac{1}{2 M} x(n) \quad \text { for } n \geq n_{2} .
$$

On the other hand, we know from [4, Lemma 1.5] (also see [16, Lemma 1]) that

$$
\liminf _{n \rightarrow \infty} \sum_{j=\tau(n)}^{n-1} p(j)=\liminf _{n \rightarrow \infty} \sum_{j=h(n)}^{n-1} p(j)
$$

Since $h(n) \geq \tau(n)$ and $(x(n))$ is non-increasing, by (1) and (25) we have

$$
\Delta x(n)+\frac{1}{2 M} p(n) x(h(n)) \leq 0, \quad n \geq n_{3} .
$$

Also, from (22) and (26), it follows that there exists a constant $c>0$ such that

$$
\sum_{j=h(n)}^{n} p(j) \geq \sum_{j=h(n)}^{n-1} p(j) \geq c>\frac{M}{\mathrm{e}}, \quad n \geq n_{3} \geq n_{2} .
$$

So, from (28), there exists an integer $n^{*} \in(h(n), n)$, for all $n \geq n_{3}$ such that

$$
\sum_{j=h(n)}^{n^{*}} p(j)>\frac{M}{2 \mathrm{e}} \text { and } \sum_{j=n^{*}}^{n} p(j)>\frac{M}{2 \mathrm{e}} .
$$

Summing up (27) from $h(n)$ to $n^{*}$ and using $(x(n))$ is non-increasing, then we have

$$
x\left(n^{*}+1\right)-x(h(n))+\frac{1}{2 M} \sum_{j=h(n)}^{n^{*}} p(j) x(h(j)) \leq 0
$$

or

$$
x\left(n^{*}+1\right)-x(h(n))+\frac{1}{2 M} x\left(h\left(n^{*}\right)\right) \sum_{j=h(n)}^{n^{*}} p(j) \leq 0 .
$$

Thus, by (29), we have

$$
-x(h(n))+\frac{1}{2 M} x\left(h\left(n^{*}\right)\right) \frac{M}{2 \mathrm{e}}<0 .
$$


Summing (27) from $n^{*}$ to $n$ and using the same facts, we get

$$
x(n+1)-x\left(n^{*}\right)+\frac{1}{2 M} \sum_{j=n^{*}}^{n} p(j) x(h(j)) \leq 0 .
$$

Thus, by (29), we have

$$
-x\left(n^{*}\right)+\frac{1}{2 M} x(h(n)) \frac{M}{2 \mathrm{e}}<0 .
$$

Combining the inequalities (30) and (31), we obtain

$$
x\left(n^{*}\right)>x(h(n)) \frac{1}{4 \mathrm{e}}>x\left(h\left(n^{*}\right)\right)\left(\frac{1}{4 \mathrm{e}}\right)^{2},
$$

and hence we have

$$
\frac{x\left(h\left(n^{*}\right)\right)}{x\left(n^{*}\right)}<(4 \mathrm{e})^{2} \quad \text { for } n \geq n_{4} .
$$

Let

$$
w=\liminf _{n \rightarrow \infty} \frac{x\left(h\left(n^{*}\right)\right)}{x\left(n^{*}\right)} \geq 1,
$$

and because of $1 \leq w \leq(4 \mathrm{e})^{2}, w$ is finite.

Now dividing (1) with $x(n)$ and then summing up from $h(n)$ to $n-1$, we obtain

$$
\sum_{j=h(n)}^{n-1} \frac{\Delta x(j)}{x(j)}+\sum_{j=h(n)}^{n-1} p(j) \frac{f(x(\tau(j)))}{x(j)}=0 .
$$

It is well known that

$$
\ln \left(\frac{x(n)}{x(h(n))}\right) \leq \sum_{j=h(n)}^{n-1} \frac{\Delta x(j)}{x(j)}
$$

So, by (33) and (34), we have

$$
\ln \left(\frac{x(n)}{x(h(n))}\right)+\sum_{j=h(n)}^{n-1} p(j) \frac{f(x(\tau(j)))}{x(\tau(j))} \frac{x(\tau(j))}{x(j)} \leq 0 .
$$

Since $h(n) \geq \tau(n)$ and $(x(n))$ is non-increasing, we get

$$
\ln \left(\frac{x(h(n))}{x(n)}\right) \geq \sum_{j=h(n)}^{n-1} p(j) \frac{f(x(\tau(j)))}{x(\tau(j))} \frac{x(h(j))}{x(j)} .
$$

Taking lower limits on both of (35) and using (21), (22) and (32), we obtain $\ln (w)>\frac{w}{\mathrm{e}}$. But this is impossible since $\ln (x) \leq \frac{x}{\mathrm{e}}$ for all $x>0$. 
Now, we consider the case where $M=0$. In this case, it is clear that by (21), we have

$$
\lim _{x \rightarrow 0} \frac{x}{f(x)}=0
$$

Since $\frac{x}{f(x)}>0$, by (36), for sufficiently large integers, we get

$$
\frac{x}{f(x)}<\varepsilon
$$

and

$$
\frac{f(x)}{x}>\frac{1}{\varepsilon}
$$

where $\varepsilon>0$ is an arbitrary real number. Thus, since $\tau(n) \leq h(n)$ and $(h(n))$ is nondecreasing, by (1) and (37), we have

$$
\Delta x(n)+\frac{1}{\varepsilon} p(n) x(h(n))<0, \quad n \geq n_{1}
$$

Summing up (38) from $h(n)$ to $n$, we obtain

$$
x(n+1)-x(h(n))+\frac{1}{\varepsilon} \sum_{j=h(n)}^{n} p(j) x(h(j))<0,
$$

and so, we get

$$
-x(h(n))+\frac{1}{\varepsilon} x(h(n)) \sum_{j=h(n)}^{n} p(j)<0 .
$$

Thus, by (28) and (39), we can write

$$
\frac{c}{\varepsilon}<1
$$

or

$$
\varepsilon>c .
$$

This contradicts $\lim _{x \rightarrow 0} \frac{x}{f(x)}=0$. The proof of the theorem is completed.

Theorem 2 Assume that (2), (3), (24) and (21) hold with $0<M<\infty$. If $(\tau(n))$ is nonmonotone, and

$$
\limsup _{n \rightarrow \infty} \sum_{j=h(n)}^{n} p(j)>\theta M
$$

where $h(n)$ is defined by (20) and $\theta>1$ is a constant, then all solutions of Eq. (1) oscillate. 
Proof Assume, for the sake of contradiction, that there exists a nonoscillatory solution $(x(n))$ of (1). In view of (24), we know from the proof of Theorem 1 that $\lim _{n \rightarrow \infty} x(n)=0$ for $n \geq n_{1}$.

On the other hand, by (21) and for every $\theta>1$, there exists a $\delta>0$ such that

$$
\frac{x}{f(x)} \leq \theta M \quad \text { for }|x|<\delta .
$$

Since $x(n) \rightarrow 0$ as $n \rightarrow \infty$, we can find a $n_{2}$ such that $0<x(n)<\delta$ for $n \geq n_{2}$, which yields

$$
\frac{x(n)}{f(x(n))} \leq \theta M
$$

or equivalently

$$
f(x(n)) \geq \frac{1}{\theta M} x(n) \quad \text { for } n \geq n_{2} .
$$

From Eqs. (1) and (41), we get

$$
\Delta x(n)+\frac{1}{\theta M} p(n) x(\tau(n)) \leq 0 .
$$

Since $h(n) \geq \tau(n)$ and $(x(n))$ is non-increasing, we obtain

$$
\Delta x(n)+\frac{1}{\theta M} p(n) x(h(n)) \leq 0 .
$$

Summing up (42) from $h(n)$ to $n$, and using the fact that $(h(n))$ is non-decreasing

$$
x(n+1)-x(h(n))+\frac{1}{\theta M} \sum_{j=h(n)}^{n} p(j) x(h(j)) \leq 0
$$

or

$$
-x(h(n))+\frac{1}{\theta M} x(h(n)) \sum_{j=h(n)}^{n} p(j)<0 .
$$

This implies

$$
-x(h(n))\left[1-\frac{1}{\theta M} \sum_{j=h(n)}^{n} p(j)\right]<0 \quad \text { for } n \geq n_{2}
$$

and hence

$$
\sum_{j=h(n)}^{n} p(j)<\theta M .
$$

Therefore, we obtain

$$
\limsup _{n \rightarrow \infty} \sum_{j=h(n)}^{n} p(j) \leq \theta M .
$$

This is a contradiction to (40). The proof is completed. 
Now, assume that $f$ is non-decreasing function, then we have the following result.

Theorem 3 Assume that (2), (3), (24) and (21) hold with $0<M<\infty$. Iff is non-decreasing, $(\tau(n))$ is non-monotone and

$$
\limsup _{n \rightarrow \infty} \sum_{j=h(n)}^{n} p(j)>M
$$

where $h(n)$ is defined by (20), then all solutions of Eq. (1) oscillate.

Proof Assume, for the sake of contradiction, that there exists a nonoscillatory solution $(x(n))$ of (1). In view of (24), we know from the proof of Theorem 1 that $\lim _{n \rightarrow \infty} x(n)=0$ for $n \geq n_{1}$.

Since $\tau(n) \leq h(n),(x(n))$ is non-increasing and $(h(n)), f$ are non-decreasing, for Eq. (1), we have

$$
\Delta x(n)+p(n) f(x(h(n))) \leq 0 .
$$

Summing up (44) from $h(n)$ to $n$, we get

$$
x(n+1)-x(h(n))+\sum_{j=h(n)}^{n} p(j) f(x(h(j))) \leq 0
$$

or

$$
-x(h(n))+f(x(h(n))) \sum_{j=h(n)}^{n} p(j)<0
$$

and so

$$
-x(h(n))\left[1-\frac{f(x(h(n)))}{x(h(n))} \sum_{j=h(n)}^{n} p(j)\right]<0 .
$$

Therefore

$$
\frac{f(x(h(n)))}{x(h(n))} \sum_{j=h(n)}^{n} p(j)<1
$$

and hence, we have

$$
\limsup _{n \rightarrow \infty} \sum_{j=h(n)}^{n} p(j) \leq M
$$

This is a contradiction to (43). The proof is completed.

Remark 1 We remark that if $(\tau(n))$ is non-decreasing, then we have $\tau(n)=h(n)$ for all $n \in \mathbb{N}$. Therefore, the condition (40) in Theorem 2 and the condition (43) in Theorem 3, 
respectively, reduce to

$$
\limsup _{n \rightarrow \infty} \sum_{j=\tau(n)}^{n} p(j)>\theta M
$$

and

$$
\limsup _{n \rightarrow \infty} \sum_{j=\tau(n)}^{n} p(j)>M
$$

Now, we present an example to show the significance of our results.

Example 1 Consider the nonlinear delay difference equation

$$
\Delta x(n)+\frac{1}{\mathrm{e}} x(\tau(n)) \ln (10+|x(\tau(n))|)=0, \quad n \geq 0,
$$

where

$$
\tau(n)= \begin{cases}n-1 & \text { if } n \in[3 k, 3 k+1], \\ -3 n+12 k+3 & \text { if } n \in[3 k+1,3 k+2], \quad k \in \mathbb{N}_{0} . \\ 5 n-12 k-13 & \text { if } n \in[3 k+2,3 k+3],\end{cases}
$$

By (20), we see that

$$
h(n):=\max _{s \leq n} \tau(s)= \begin{cases}n-1 & \text { if } n \in[3 k, 3 k+1], \\ 3 k & \text { if } n \in[3 k+1,3 k+2.6], \quad k \in \mathbb{N}_{0} . \\ 5 n-12 k-13 & \text { if } n \in[3 k+2.6,3 k+3],\end{cases}
$$

If we put $p(n)=\frac{1}{\mathrm{e}}$ and $f(x)=x \ln (10+|x|)$. Then we have

$$
M=\limsup _{x \rightarrow 0} \frac{x}{f(x)}=\limsup _{x \rightarrow 0} \frac{x}{x \ln (10+|x|)}=\frac{1}{\ln (10)}
$$

and

$$
\liminf _{n \rightarrow \infty} \sum_{j=\tau(n)}^{n-1} p(j)=\frac{1}{\mathrm{e}}>\frac{M}{\mathrm{e}}=\frac{1}{\mathrm{e} \ln (10)},
$$

that is, all conditions of Theorem 1 are satisfied and therefore all solutions of (47) oscillate.

Competing interests

The authors declare that they have no competing interests.

Authors' contributions

All authors contributed equally to the manuscript. All the authors read and approved the final manuscript.

\section{Author details}

${ }^{1}$ Department of Mathematics, Faculty of Science, Akdeniz University, Antalya, Turkey. ${ }^{2}$ Department of Mathematics,

Faculty of Science and Arts, Afyon Kocatepe University, Afyonkarahisar, Turkey. 


\section{Publisher's Note}

Springer Nature remains neutral with regard to jurisdictional claims in published maps and institutional affiliations.

Received: 27 February 2018 Accepted: 2 May 2018 Published online: 08 May 2018

\section{References}

1. Agarwal, R.P.: Difference Equations and Inequalities. Marcel Dekker, New York (2000)

2. Agarwal, R.P., Grace, S.R., O'Regan, D.: Oscillation Theory for Difference and Functional Differential Equations. Kluwer Academic, The Netherlands (2000)

3. Chatzarakis, G.E., Koplatadze, R., Stavroulakis, I.P.: Oscillation criteria of first order linear difference equations with delay argument. Nonlinear Anal. 68, 994-1005 (2008)

4. Chatzarakis, G.E., Koplatadze, R., Stavroulakis, I.P.: Optimal oscillation criteria for first order difference equations with delay argument. Pac. J. Math. 235, 15-33 (2008)

5. Chatzarakis, G.E., Philos, Ch.G., Stavroulakis, I.P.: On the oscillation of the solutions to linear difference equations with variable delay. Electron. J. Differ. Equ. 2008, 50 (2008)

6. Chatzarakis, G.E., Philos, Ch.G., Stavroulakis, I.P.: An oscillation criterion for linear difference equations with general delay argument. Port. Math. 66(4), 513-533 (2009)

7. Chen, M.-P., Yu, J.S.: Oscillations of delay difference equations with variable coefficients. In: Proceedings of the First International Conference on Difference Equations, pp. 105-114. Gordon and Breach, London (1994)

8. Erbe, L.H., Zhang, B.G.: Oscillation of discrete analogues of delay equations. Differ. Integral Equ. 2, 300-309 (1989)

9. Györi, I., Ladas, G.: Linearized oscillations for equations with piecewise constant arguments. Differ. Integral Equ. 2, 123-131 (1989)

10. Györi, I., Ladas, G.: Oscillation Theory of Delay Differential Equations with Applications. Clarendon Press, Oxford (1991)

11. Jiang, J.C., Tang, X.H.: Oscillation of nonlinear delay difference equations. J. Comput. Appl. Math. 146, 395-404 (2002)

12. Ladas, G., Philos, Ch.G., Sficas, Y.G.: Sharp conditions for the oscillation of delay difference equations. J. Appl. Math. Simul. 2, 101-111 (1989)

13. Ladas, G.: Explicit conditions for the oscillation of difference equations. J. Math. Anal. Appl. 153, 276-287 (1990)

14. Ladde, G.S., Lakshmikantham, V., Zhang, B.G.: Oscillation Theory of Differential Equations with Deviating Arguments. Marcel Dekker, New York (1987)

15. Philos, Ch.G.: On oscillations of some difference equations. Funkc. Ekvacioj 34, 157-172 (1991)

16. Öcalan, Ö:: An improved oscillation criterion for first order difference equations. Bull. Math. Soc. Sci. Math. Roum. 59(107)(1), 65-73 (2016)

17. Tang, X.H., Yu, J.S.: Oscillation of nonlinear delay difference equations. J. Math. Anal. Appl. 249, 476-490 (2000)

18. Yan, W., Meng, Q., Yan, J.: Oscillation criteria for difference equation of variable delays. Dyn. Contin. Discrete Impuls. Syst., Ser. A Math. Anal. 13A, 641-647 (2006)

19. Yu, J.S., Wang, Z.C.: Some further results on oscillation of neutral differential equations. Bull. Aust. Math. Soc. 46 , 149-157 (1992)

20. Zhang, B.G., Tian, C.J.: Oscillation criteria for difference equations with unbounded delay. Comput. Math. Appl. 35(4), 19-26 (1998)

21. Zhang, B.G., Tian, C.J.: Nonexistence and existence of positive solutions for difference equations with unbounded delay. Comput. Math. Appl. 36, 1-8 (1998)

22. Zhang, B.G., Yan, X.Z., Liu, X.Y.: Oscillation criteria of certain delay dynamic equations on time scales. J. Differ. Equ. Appl. 11(10), 933-946 (2005)

23. Zhou, Y.: Oscillation of higher-order delay difference equations. Adv. Differ. Equ. 2006, Article ID 65789 (2006)

\section{Submit your manuscript to a SpringerOpen ${ }^{\circ}$ journal and benefit from:}

- Convenient online submission

- Rigorous peer review

- Open access: articles freely available online

- High visibility within the field

- Retaining the copyright to your article

Submit your next manuscript at $\gg$ springeropen.com 